\title{
乌s \\ Full three-dimensional approach to the design and simulation of a radio-frequency quadrupole
}

\author{
B. Mustapha, ${ }^{*}$ A. A. Kolomiets, ${ }^{\dagger}$ and P. N. Ostroumov \\ Physics Division, Argonne National Laboratory, Argonne, Illinois 60439, USA
}

(Received 25 September 2013; published 6 December 2013)

\begin{abstract}
We have developed a new full 3D approach for the electromagnetic and beam dynamics design and simulation of a radio-frequency quadrupole (RFQ). A detailed full 3D model including vane modulation was simulated, which was made possible by the ever advancing computing capabilities. The electromagnetic (EM) design approach was first validated using experimental measurements on an existing prototype RFQ and more recently on the actual full size RFQ. Two design options have been studied, the original with standard sinusoidal modulation over the full length of the RFQ; in the second design, a trapezoidal modulation was used in the accelerating section of the RFQ to achieve a higher energy gain for the same power and length. A detailed comparison of both options is presented supporting our decision to select the trapezoidal design. The trapezoidal modulation increased the shunt impedance of the RFQ by $34 \%$, the output energy by $15 \%$ with a similar increase in the peak surface electric field, but practically no change in the dynamics of the accelerated beam. The beam dynamics simulations were performed using three different field methods. The first uses the standard eight-term potential to derive the fields, the second uses 3D fields from individual cell-by-cell models, and the third uses the 3D fields for the whole RFQ as a single cavity. A detailed comparison of the results from TRACK shows a very good agreement, validating the 3D fields approach used for the beam dynamics studies. The EM simulations were mainly performed using the CST MICROWAVE-STUDIO with the final results verified using other software. Detailed segmentby-segment and full RFQ frequency calculations were performed and compared to the measured data. The maximum frequency deviation is about $100 \mathrm{kHz}$. The frequencies of higher-order modes have also been calculated and finally the modulation and tuners effects on both the frequency and field flatness have been studied. We believe that with this new full 3D approach, the enhanced computing capabilities and the calculation precision the electromagnetic design software offer, we may be able to skip the prototyping phase and build the final product at once, although we recognize that prototyping is still needed to establish and validate the fabrication procedure.
\end{abstract}

DOI: 10.1103/PhysRevSTAB.16.120101

PACS numbers: 29.20.Ej, 29.27.Bd

\section{INTRODUCTION}

Since its invention by Kapchinsky and Teplyakov [1], the radio-frequency quadrupole (RFQ) has become an essential part of proton and heavy-ion accelerators. Besides ITEP-Protvino [2] and ITEP-Moscow [3], significant work has been done on RFQs at Los Alamos National Laboratory [4], CERN [5], and many other labs in both software and hardware. Design and simulation codes have been developed as well as significant development in engineering and fabrication. In this paper, we make the attempt of complementing this work by the development of a new full 3D design and simulation approach that was successfully applied to the design and simulation of the ATLAS upgrade RFQ [6]. The RFQ is now being used for

\footnotetext{
*contact author: brahim@anl.gov
}

${ }^{\dagger}$ visiting from ITEP-Moscow

Published by the American Physical Society under the terms of the Creative Commons Attribution 3.0 License. Further distribution of this work must maintain attribution to the author(s) and the published article's title, journal citation, and DOI. routine ATLAS operations and has demonstrated excellent performance $[7,8]$.

The beam dynamics and the electromagnetic design of a RFQ are usually done separately using different tools. A code such as PARMTEQ [9] or DesRFQ [10] is used for the beam dynamics design and a code such as SUPERFISH [11], MAFIA [12] or MICROWAVE-STUDIO (MWS) from CST [13] is used for the electromagnetic design. Because of limited computing capabilities in the past, full 3D modeling of a few-meter-long RFQ was not possible. Often a short segment is used for these simulations that needs to be verified by building a prototype first. For the same reasons, vane modulations are often ignored due to the complication and the extreme level of detail they introduce to the geometry. In the new full 3D approach developed in this work, we have modeled the full five-segment 4-m long ATLAS upgrade RFQ [6] in MWS. The actual vane modulations and the tuners were also included to study their effects on both the frequency and field distribution. We were also able to produce 3D field maps to use for beam dynamics simulations. Verifying the beam dynamics using 3D fields from the electromagnetic design software provides a more consistent way for design evaluation. 
After presenting the RFQ design goals and approach, we review the design considerations and the studies performed to help us decide on the best design. The beam dynamics design and simulation section presents a benchmark of the full 3D field approach and a detailed comparison between the sinusoidal and trapezoidal design options of the RFQ. The electromagnetic design section compares the calculated frequencies to the measured values and presents the effects of modulation and tuners on the frequency and field flatness. A comparison of the peak surface electric fields between the sinusoidal and trapezoidal designs is presented as well.

\section{DESIGN CONSIDERATIONS}

During the design of the low $\mathrm{q} / \mathrm{m}$ continuous wave (CW) $60.625 \mathrm{MHz}$ RFQ for the ATLAS efficiency and intensity upgrade [14], we encountered several choices and decisions to make. Among these choices were (i) the vane shape: a full-vane design or a split-coaxial design; (ii) the RFQ length and number of segments for power and cooling considerations; (iii) external or internal bunching; (iv) trapezoidal or sinusoidal modulation in the accelerating section of the RFQ; and (v) matching to the following superconducting linac.

\section{A. Full-vane versus split-coaxial design}

Figure 1 shows two design options for the same $60 \mathrm{MHz}$ RFQ. The first is a full-vane design and the second with holes or windows cut into the vanes known as the splitcoaxial design. A long structure could be built in segments or modules. In the split-coaxial design, every individual segment is a split-coaxial structure where the inner conductor is split into four electrodes alternately attached to either the input or output end of the outer conductor.
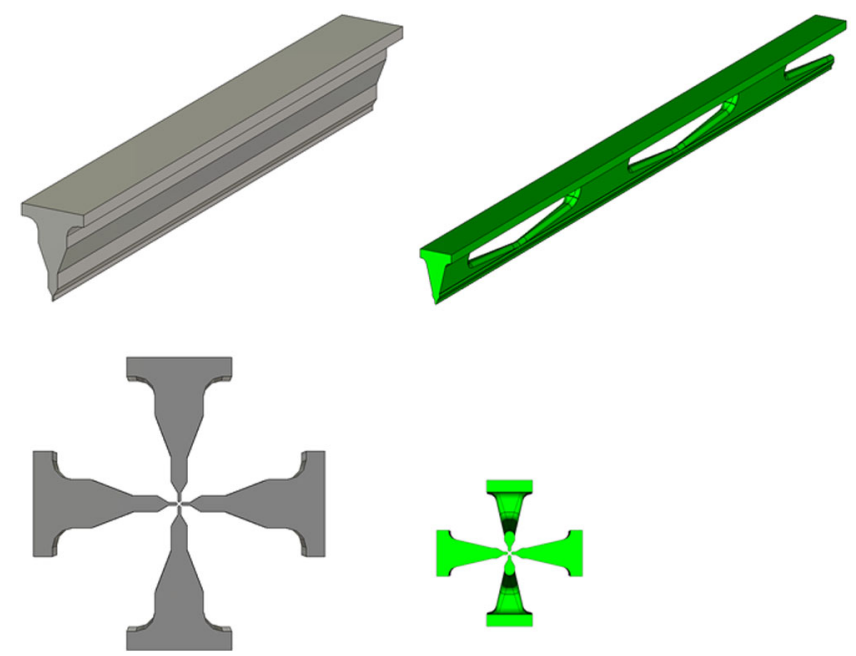

FIG. 1. Two design options for the RFQ vane. The first is a full vane (left) and the second has windows (right). The bottom plots show cross sections for both options scaled to the actual size. The transverse size is reduced from about $1 \mathrm{~m}$ to $0.5 \mathrm{~m}$.
TABLE I. Comparison of important parameters between the full-vane and the window-coupled designs.

\begin{tabular}{lcc}
\hline \hline Parameter & Full vane & Split coaxial \\
\hline Transverse size $(\mathrm{cm})$ & 109 & 54 \\
Power $(\mathrm{kW})$ & 23 & 52 \\
Nearest mode separation $(\mathrm{MHz})$ & -1.5 & +10.5 \\
\hline \hline
\end{tabular}

The segments are assembled back to back and are strongly coupled by the magnetic flux through the alternating windows formed by the neighboring segments. The splitcoaxial structure was first proposed by Muller at GSI [15] and a multimodule or multisegment version was first implemented by Arai et al. [16] for an 8-m long lowfrequency RFQ. The actual window-coupled design is mainly based on the 4-ladder structure proposed by Andreev [17].

The split-coaxial or window-coupled design is strongly coupled and should be more stable with good mode separation, which makes it less sensitive to tuning and manufacturing errors. It does, however, require more power because the rf current density is higher. Another argument in favor of the window-coupled design is the reduced transverse size compared to the full-vane design, as shown in the bottom of Fig. 1. This feature is of particular importance at low frequency - the suggested cutoff would be around $100 \mathrm{MHz}$, below which the RFQ transverse size becomes cumbersome, affecting both the cost and the handling of the structure.

Table I gives a comparison of the important parameters discussed above for the two design options. The full-vane design is about twice as large but requiring half the power of the window-coupled design. We also notice that the nearest dipole mode for the full-vane design is separated by only $1.5 \mathrm{MHz}$ from the main mode. A close dipole mode with a lower frequency than the main mode complicates the tuning and may lead to the superposition of the dipole and quadrupole fields. For the window-coupled design, however, the closest mode is more than $10 \mathrm{MHz}$ above the main mode and should not interfere with the tuning and operation of the RFQ.

\section{B. RFQ length and power management}

Power management is a very critical issue for a $\mathrm{CW}$ RFQ and should be carefully addressed from the start. The original ATLAS RFQ design was four segments spanning $3 \mathrm{~m}$ with $92 \mathrm{kV}$ vane voltage. The thermal and structural analysis of this original design [18] showed that significant modifications of the cooling channels are required that would affect the original manufacturing plan and increase the cost of the device. Instead, we opted to lengthen the $\mathrm{RFQ}$, hence reducing the required voltage and power. For the same output energy, we expect the vane voltage to reduce linearly with the number of segments and the power 
TABLE II. Comparison between the original four-segment and final five-segment designs for the ATLAS upgrade RFQ.

\begin{tabular}{lcc}
\hline \hline Parameter & Four-segment & Five-segment \\
\hline Vane length $(\mathrm{cm})$ & 305 & 381 \\
Voltage $(\mathrm{kV})$ & 92 & 70 \\
Power $(\mathrm{kW})$ & 72 & 52 \\
Peak temperature $\left({ }^{\circ} \mathrm{C}\right)$ & 77 & 27 \\
Vane displacement $($ inch) & 0.013 & 0.0023 \\
\hline \hline
\end{tabular}

density (power per segment) to reduce quadratically. In addition to increasing the length of the RFQ to lower the power density, the locations and paths of the cooling channels were adjusted to reduce vane displacements and ensure better frequency control with temperature. The results of the thermal analysis for the final design can be found in [19]. Table II shows a comparison between the original four-segment design and the final five-segment design for the ATLAS upgrade RFQ. The results confirm the expected voltage and power reduction. The reductions in the peak temperature and vane displacement are also due to the redesign of the cooling channels.

Adding a fifth segment does increase the cost and footprint of the RFQ, but it significantly reduces the risk of excessive heating and simplifies power management; ensuring high-reliability $\mathrm{CW}$ operation.

\section{External versus internal bunching}

For a RFQ, the initial beam bunching could be done internally inside the RFQ [20] or externally upstream of the RFQ [21]. In the latter, a significantly smaller longitudinal emittance could be formed at the expense of losing a fraction of the beam that could reach $20 \%$ [22]. The RFQ design and the upstream low-energy beam transport depend on this choice. The external bunching could be done using a multiharmonic buncher followed by a drift space upstream of the RFQ. This requires more space in the lowenergy beam transport but shortens the RFQ by eliminating the prebunching section. The smaller longitudinal emittance obtained by prebunching makes the injection to and the tuning of the subsequent linac much smoother and reduces the risk of beam loss at higher energies [23]. For the case of ATLAS, a four-harmonic buncher is already in use to bunch the beam into the positive ion injector and will be used with the RFQ upgrade.

\section{Input and output radial matchers}

While an input radial matcher is required to match the incoming larger beam radius to the strong focusing and smaller beam radius inside the RFQ, an output matcher is not required. An end cell also called a fringe field cell is often used [24]. In our case the RFQ is followed by axialsymmetric solenoid focusing in the first positive ion injector cryostat. The matching could be done either by external quadrupoles or internally by adding an output
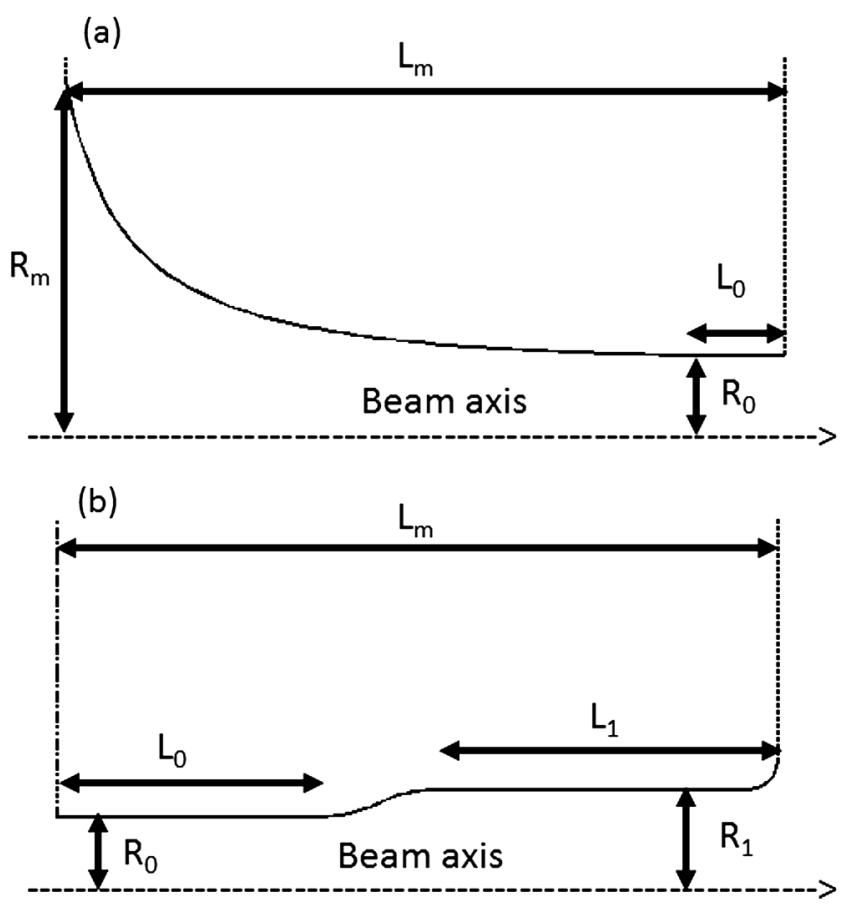

FIG. 2. Geometry of a standard input matcher (a) and the geometry of the new type of output matcher (b).

radial matcher at the end of the RFQ. The second option is more desirable to conserve space and shorten the beam path between the RFQ and the first cryomodule. In this work, instead of the more standard output matcher geometry obtained by mirroring the input radial matcher [25] shown in Fig. 2(a), we have developed a new type of output matcher with the geometry shown in Fig. 2(b). The new design is more compact with the geometric parameters optimized to obtain an axial-symmetric beam at the RFQ exit. More details on the procedure can be found in [26].

\section{E. Trapezoidal versus sinusoidal modulation}

In the accelerating section of a RFQ, the bunch is already formed and more efficient acceleration could be obtained by replacing the standard sinusoidal modulation by a trapezoidal one as demonstrated in the original work by Belyaev et al. [27]. The trapezoidal modulation produces a more peaked accelerating field which when synchronized with the beam bunch leads to a much higher transit-time factor and higher energy gain [26]. However, the narrower geometric curvature in the trapezoidal modulation, as shown in Fig. 3, could affect both the peak surface electric field and the beam focusing in the accelerating section of the RFQ. Both of these issues were investigated and found to be under control, as will be discussed later. Table III shows a comparison between the original sinusoidal modulation design and the final trapezoidal modulation design for the ATLAS upgrade RFQ over the same length. We note, in particular, that the peak surface electric field remains in the feasible range, 


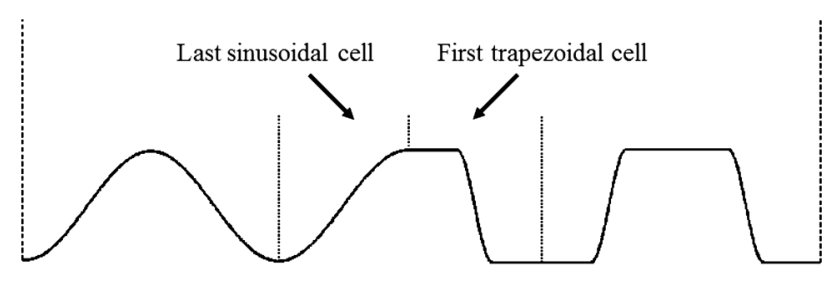

Beam axis

FIG. 3. Vane tip geometry showing the transition from sinusoidal to trapezoidal modulation. Note that the horizontal and vertical dimensions are not to scale.

below 1.8 Kilpatrick units, without optimization of the trapezoidal geometry. Optimization may be required if the peak surface field approaches or exceeds 2 Kilpatrick units. This could be done by reducing the slope of the trapeze and applying more rounding at both ends of the slope. A compromise between additional energy gain and peak surface field could be reached in this way.

Based on the design considerations discussed above, the $60 \mathrm{MHz}$ ATLAS upgrade CW RFQ is a 4-m long five-segment split-coaxial structure with a sinusoidal modulation in the bunching section and a trapezoidal one in the acceleration section. The prebunching is provided by a four-harmonic buncher placed upstream of the RFQ. The input matcher is a standard six-cell matcher. A very short output matcher $\left(0.75^{*} \beta \lambda\right)$ was specifically designed

TABLE III. Comparison between the original sinusoidal and final trapezoidal modulation design for the ATLAS upgrade RFQ for the same voltage and power.

\begin{tabular}{lcc}
\hline \hline Parameter & Sinusoidal & Trapezoidal \\
\hline Output energy $(\mathrm{keV} / \mathrm{u})$ & 262 & 296 \\
Acceleration efficiency $(\%)$ & 83 & 83 \\
Transverse emitance, $4 \times \mathrm{rms}$ & 0.84 & 0.84 \\
$\quad(\mathrm{~mm}$ mrad) & & \\
Peak surface field (Kilpatrick units) & 1.42 & 1.67 \\
\hline \hline
\end{tabular}

TABLE IV. Main parameters of the ATLAS upgrade RFQ.

\begin{tabular}{ll}
\hline \hline Parameter/feature & Value \\
\hline Input energy $(\mathrm{q} / \mathrm{m} \geq 1 / 7)$ & $30 \mathrm{keV} / \mathrm{u}$ \\
Output energy & $296 \mathrm{keV} / \mathrm{u}$ \\
Frequency & $60.625 \mathrm{MHz}$ \\
Vane voltage & $70 \mathrm{kV}$ \\
Power & $52 \mathrm{~kW}$ \\
Average radius & $7.2 \mathrm{~mm}$ \\
Length & $3.81 \mathrm{~m}$ \\
Transverse normalized acceptance & $2 \pi \mathrm{mm} \mathrm{mrad}$ \\
Longitudinal RMS emittance & $20 \pi \mathrm{deg} \mathrm{keV} / \mathrm{u}$ \\
Bunching & external \\
\hline \hline
\end{tabular}

and optimized to produce an axial-symmetric beam for direct injection into a solenoidal focusing linac. The design parameters for the ATLAS upgrade RFQ are listed in Table IV.

\section{RFQ DESIGN GOALS AND APPROACH}

The main RFQ design goals are as follows. (i) Satisfy the beam requirements for the output beam energy, emittance, and transmission. (ii) The design should have the lowest possible RF power consumption. (iii) The design target frequency should be tunable to the operational frequency using the designed tuners. (iv) Neighboring modes should be far enough from the operational mode to avoid any interference. (v) Verify the beam dynamics with more than one method, using 3D fields if possible. (vi) Produce accurate vane modulation for manufacturing.

The design of a CW RFQ is a complex process where many critical design issues need to be addressed from the start. For this purpose, a very careful design and fabrication procedure was developed. A closely interactive and iterative procedure was adopted, starting from the beam dynamics design and simulations, to the electromagnetic design, to the engineering design, and ending by manufacturing.

\section{BEAM DYNAMICS DESIGN AND SIMULATIONS}

The goal of the beam dynamics design is to produce the vane modulation to satisfy the beam requirements. As mentioned earlier, the beam is prebunched in a fourharmonic buncher before injection into the RFQ. Figure 4 shows the longitudinal beam distribution at the entrance of the RFQ. The multiharmonic buncher was simulated using realistic 3D fields with the amplitudes of the four harmonics optimized to maximize beam transmission through the RFQ. The RFQ is designed to accept the central part of the distribution containing more than $80 \%$ of the particles within a phase width of about $\pm 50 \mathrm{deg}$ at $60.625 \mathrm{MHz}$. Therefore the RFQ will not include a prebunching section

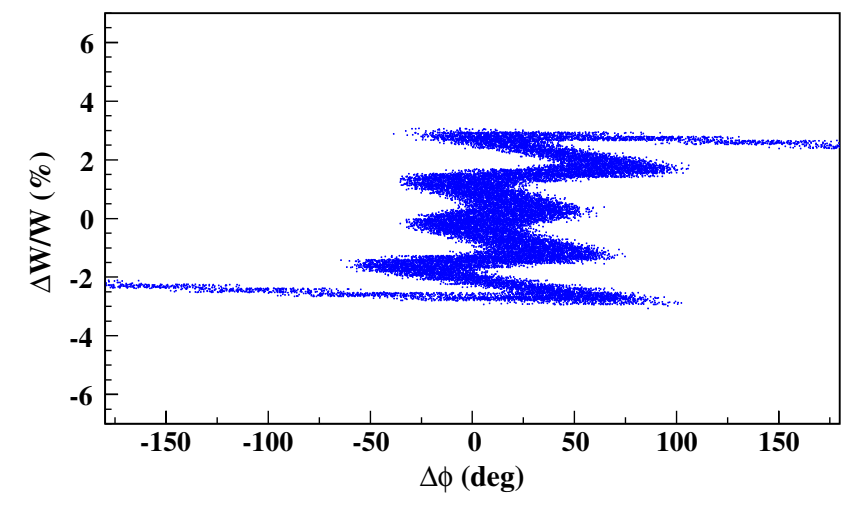

FIG. 4. The longitudinal beam distribution formed by a fourharmonic buncher at the RFQ entrance. 
and the starting phase is set to $-50 \mathrm{deg}$ instead of $-90 \mathrm{deg}$ in a conventional RFQ.

The original design with pure sinusoidal vane modulation was produced using the RFQ design code DesRFQ [10]. DesRFQ produces an output file with cell-by-cell coefficients for the eight-term potential using the FourierBessel expansion [3]. In the second design, the last 40 accelerating cells were converted from sinusoidal to trapezoidal modulations. In this case we used the 3D fields calculated using EM-Studio. The modulation factor was fixed to the maximum value of $\sim 2$, so only the length is adjusted cell by cell for a smooth synchronous phase. Figure 5 shows a comparison of the cell-by-cell RFQ parameters; modulation, synchronous phase, energy, and transverse phase advance for both designs.

Extensive beam dynamics simulations were performed for both designs. Three different field models have been used in these simulations, namely, the eight-term potential expansion, the cell-by-cell 3D fields, and the full-cavity 3D fields. In the cell-by-cell case, the cells were modeled individually in EM-Studio and the electrostatic field extracted separately for every cell. No magnetic field was included in this case, assuming the electrostatic approximation. In the full-cavity case, the whole RFQ was modeled as a single cavity in MW-Studio and the electromagnetic fields extracted in a single file for all cells. For the original sinusoidal design, the eight-term potential
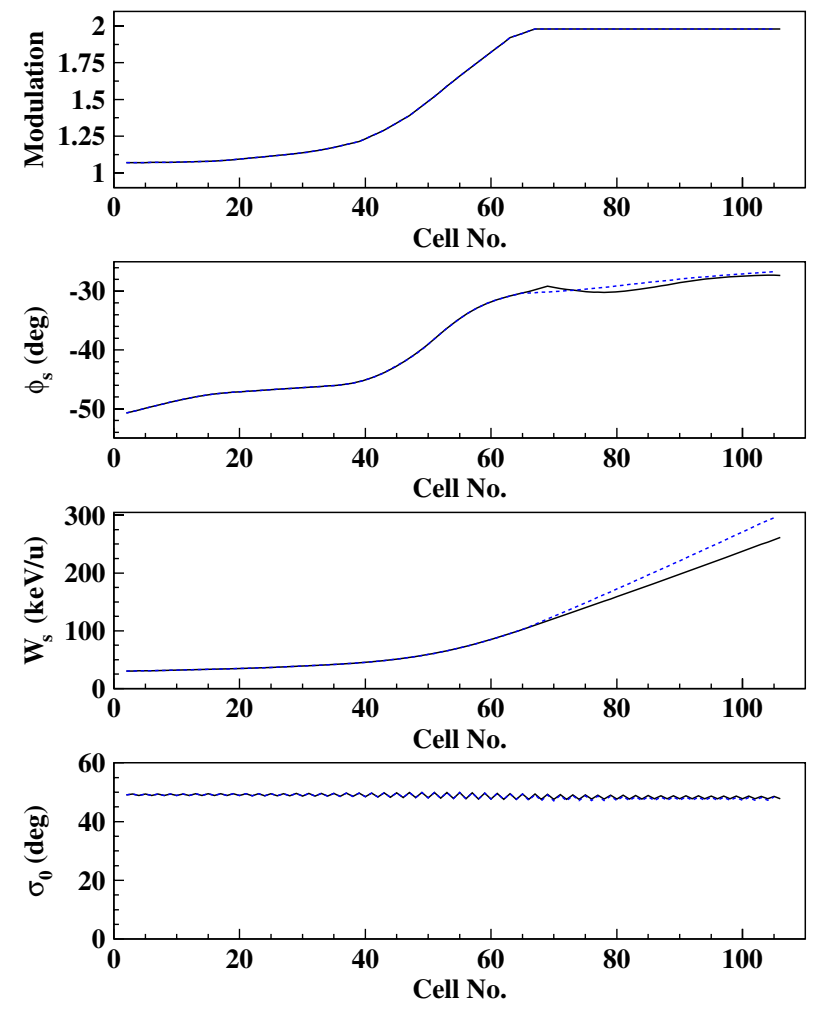

FIG. 5. Comparison of the RFQ design parameters between the original sinusoidal design (solid black) and the final trapezoidal vane modulation design (dashed blue). coefficients from DesRFQ were used to benchmark the cell-by-cell 3D fields from EM-Studio. For the final trapezoidal design, results using the cell-by-cell 3D fields were compared to those obtained with the full-cavity 3D fields extracted from MW-Studio. All the simulations were performed for a $\mathrm{q} / \mathrm{A}=1 / 7$ beam with $10^{5}$ particles and no space charge. The initial distribution is a $4 \mathrm{D}$ waterbag with a total emittance of $1.2 \pi \mathrm{mm}$ mrad $(6 \times$ the $\mathrm{rms}$ value $)$, which is larger than typical ATLAS beams. Although it was designed for zero current beams, the RFQ is capable of accelerating higher intensity beams. Experimental beam studies with currents up to $300 \mu \mathrm{A}$ and simulations with currents up to $30 \mathrm{~mA}$ were carried out successfully [7].

\section{A. Cell-by-cell 3D models versus the eight-term potential}

The eight-term potential field description is widely used and trusted for the case of sinusoidal vane modulation [28]. In order to benchmark our new full 3D design and simulation approach, we have compared the fields and beam dynamics from the cell-by-cell 3D model described above to the results obtained using the eight-term potential description. This was obviously done for the sinusoidal design option of the RFQ. Once validated, the 3D approach could be safely used for the trapezoidal design option
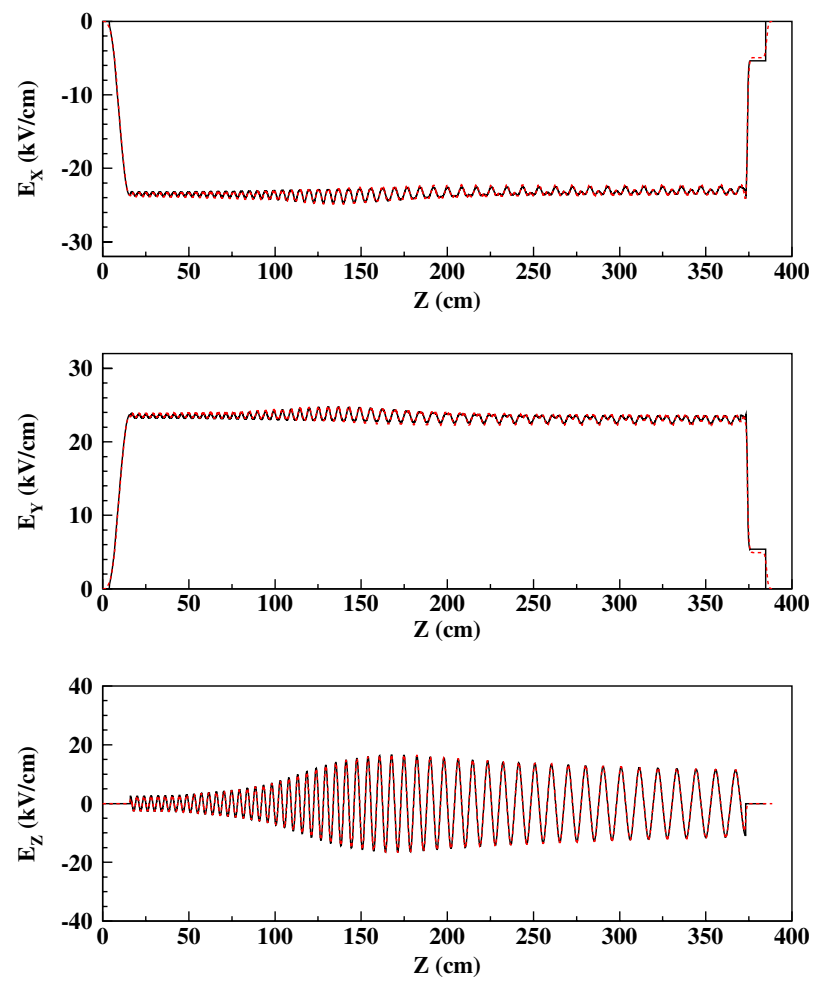

FIG. 6. Comparison of the electric field components along the RFQ at mid aperture between the eight-term potential (solid black) and the cell-by-cell 3D model (dashed red) of the original sinusoidal design. The almost perfect superposition of the curves reflects the good agreement. 

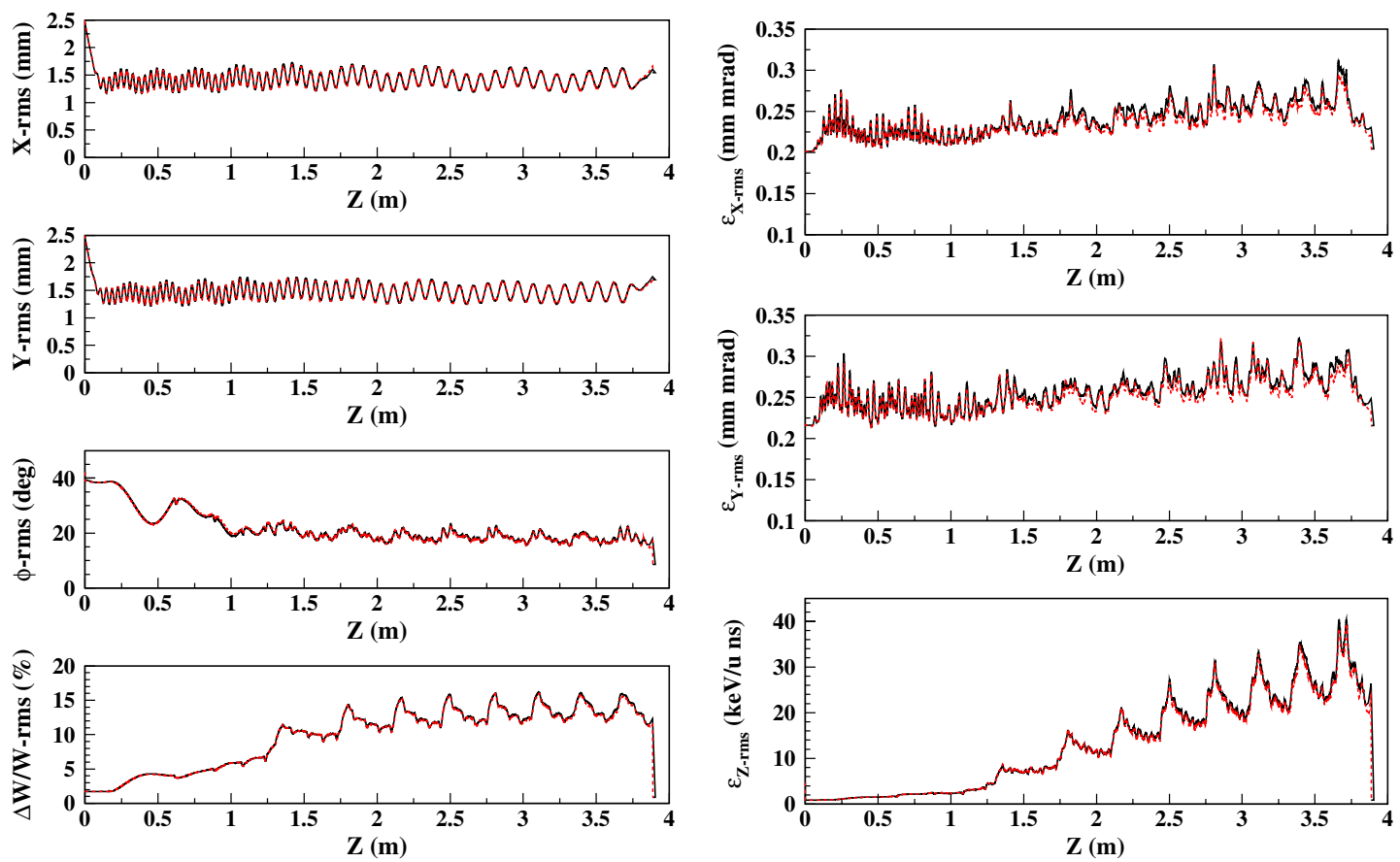

FIG. 7. Comparison between the eight-term potential (solid black) and the 3D cell-by-cell model (dashed red) for the original sinusoidal design. On the left are the rms beam envelopes and on the right the rms beam emittances along the RFQ. The curves include all particles within the aperture (accelerated or not). The peaks and valleys on the longitudinal plots correspond to oscillations of nonaccelerated particles extending the longitudinal beam tail.

for which an analytical potential formula is yet to be developed.

A detailed comparison of the cell-by-cell 3D fields to those from the eight-term potential model produced by the RFQ design code DesRFQ showed that the longitudinal component agree to better than $1 \%$ while the transverse components agree to $1 \%-2 \%$; see Fig. 6. A comparison of the beam dynamics calculated using the code TRACK [29] is shown in Fig. 7 for both the beam rms envelopes and rms emittances. An excellent agreement is observed. The curves include all particles within the aperture (accelerated or not).

\section{B. Full-cavity 3D model versus cell-by-cell model}

For the final design with trapezoidal vane modulation in the acceleration section, a full RFQ model was built in MW-Studio as a single cavity in addition to the cell-by-cell 3D models in EM-Studio. After solving the eigenmode problem and verifying the frequency, the full 3D fields were extracted on a $25 \times 25 \times 4001$ grid in the aperture area. The $E$ and $H$ fields were combined into a single-cavity field file for TRACK and used for simulations. A comparison of the $E$ field components between the cell-by-cell 3D fields from EM-Studio and the single-cavity field from MW-Studio is shown in Fig. 8. As can be seen the deviations are on the order of $1 \%-2 \%$. In TRACK, the field phase and amplitude were adjusted to get the appropriate bunching and the correct output energy. The comparison of the
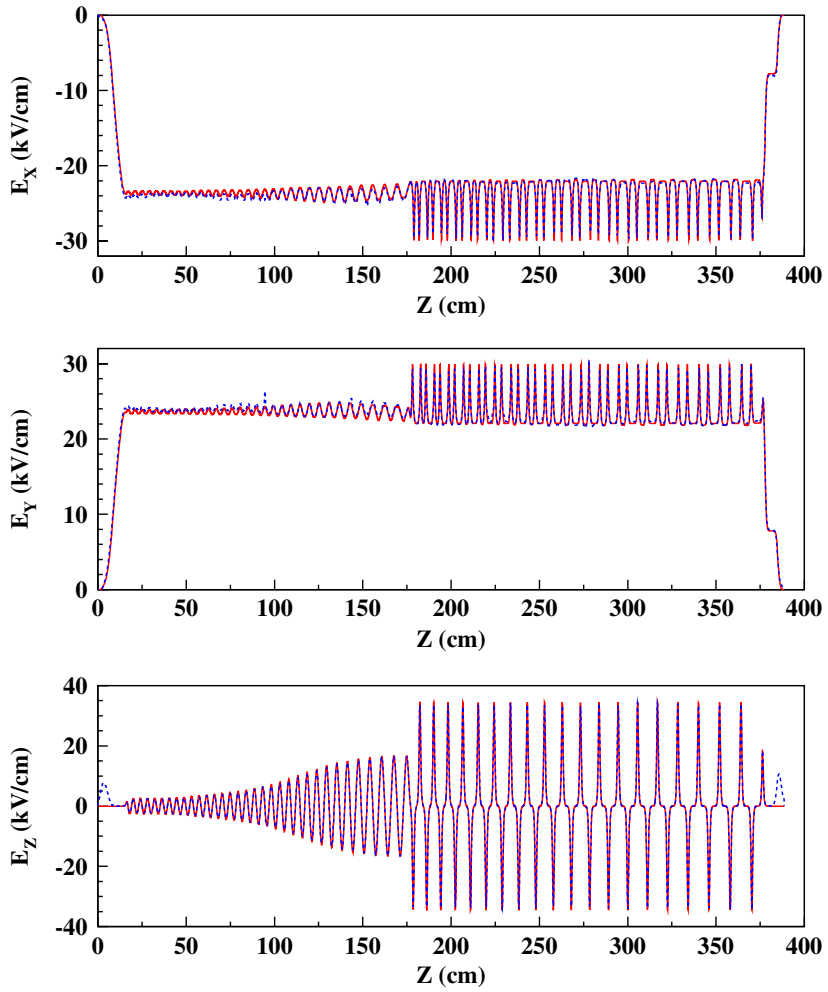

FIG. 8. Comparison of the electric field components along the RFQ at mid aperture between the cell-by-cell 3D model (solid red) and the full-cavity 3D model (dashed blue) for the final trapezoidal design. 

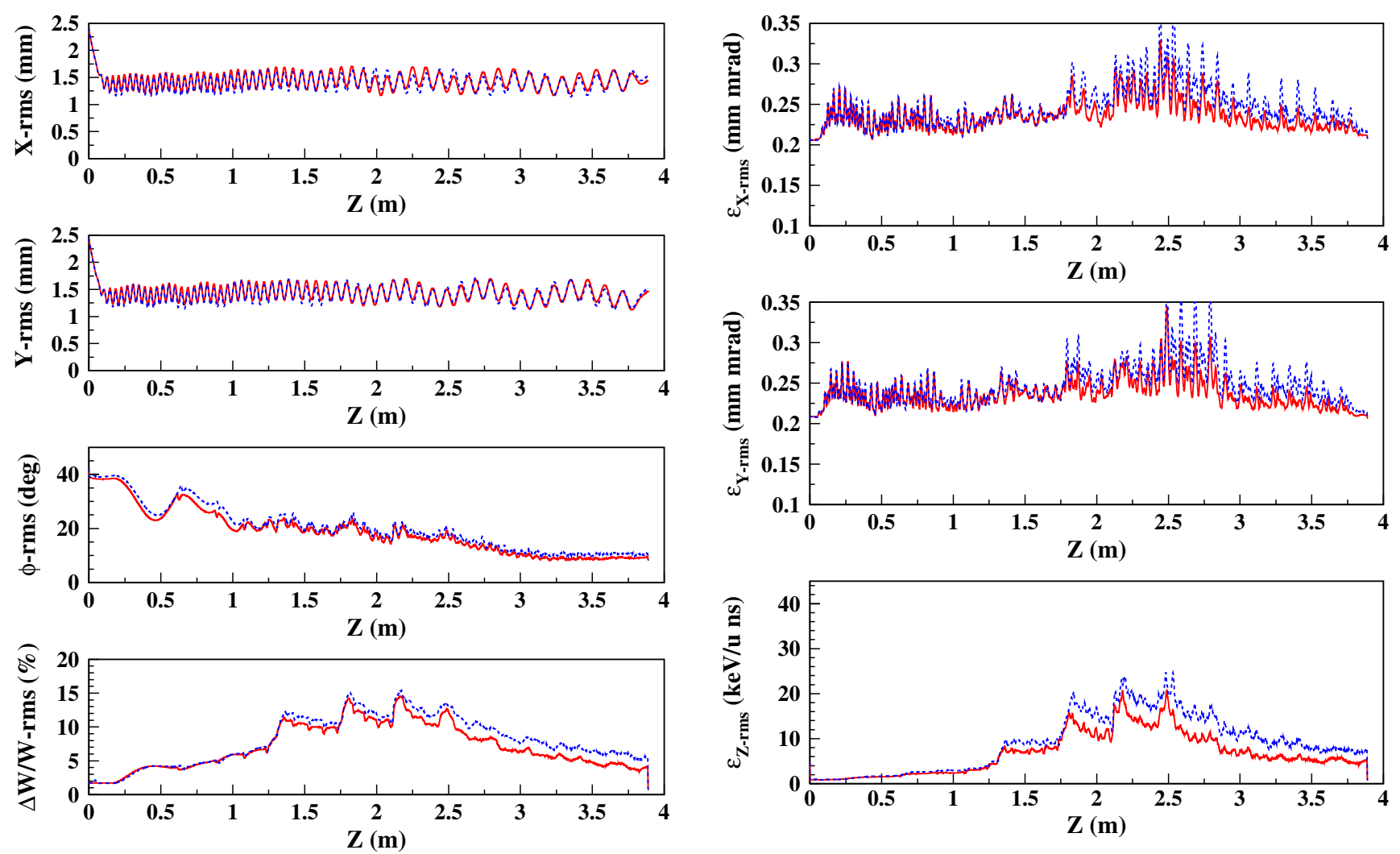

FIG. 9. Comparison between the cell-by-cell (solid red) and the full-cavity 3D models (dashed blue) for the final trapezoidal design. On the left are rms beam envelopes and on the right rms beam emittances along the RFQ. The curves include all particles within the aperture (accelerated or not). The peaks and valleys on the longitudinal plots, corresponding to oscillations of nonaccelerated particles, are present in the sinusoidal section but they disappear in the trapezoidal section of the RFQ.

beam dynamics simulations is shown in Fig. 9 for both the rms envelopes and emittances. Again a very good agreement was obtained. The minor discrepancies observed in the longitudinal plane towards the end of the RFQ may be due to the difference in accuracy between the cell-by-cell and the full-cavity 3D fields affecting the beam tail. The positive results of these comparisons constitute a validation of the overall 3D field calculation and beam dynamics simulation approach.

\section{Beam dynamics: trapezoidal versus sinusoidal}

Figure 10 shows the input beam within the acceptance of the RFQ for both design options. Realistic apertures with actual vane modulations were used in these calculations. Although very similar, we notice that the transverse acceptance for the sinusoidal design is wider. The longitudinal acceptance is practically the same because it is determined by the bunching section which is common to both designs. The calculated transverse acceptance of the RFQ exceeds $2 \pi \mathrm{mm}$ mrad while the typical ATLAS beam emittance is less than $1 \pi \mathrm{mm}$ mrad.

The comparison of beam dynamics is shown on Fig. 11 for the beam rms envelopes and emittances. No significant transverse emittance growth is observed after changing the modulation from sinusoidal to trapezoidal in the accelerating section of the RFQ, but we can see a reduction in the longitudinal beam envelopes and emittance suggesting a filtering of the low-energy tail by the trapezoidal vanes. Table $\mathrm{V}$ compares the output beam
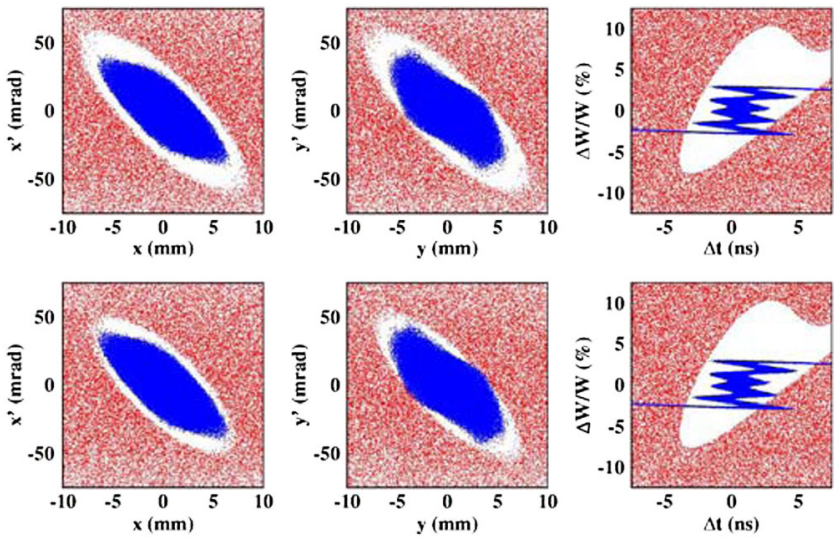

FIG. 10. Comparison of the RFQ acceptance between the original sinusoidal design (top) and the final trapezoidal vane modulation design (bottom). Realistic apertures with actual vane modulations were used. 

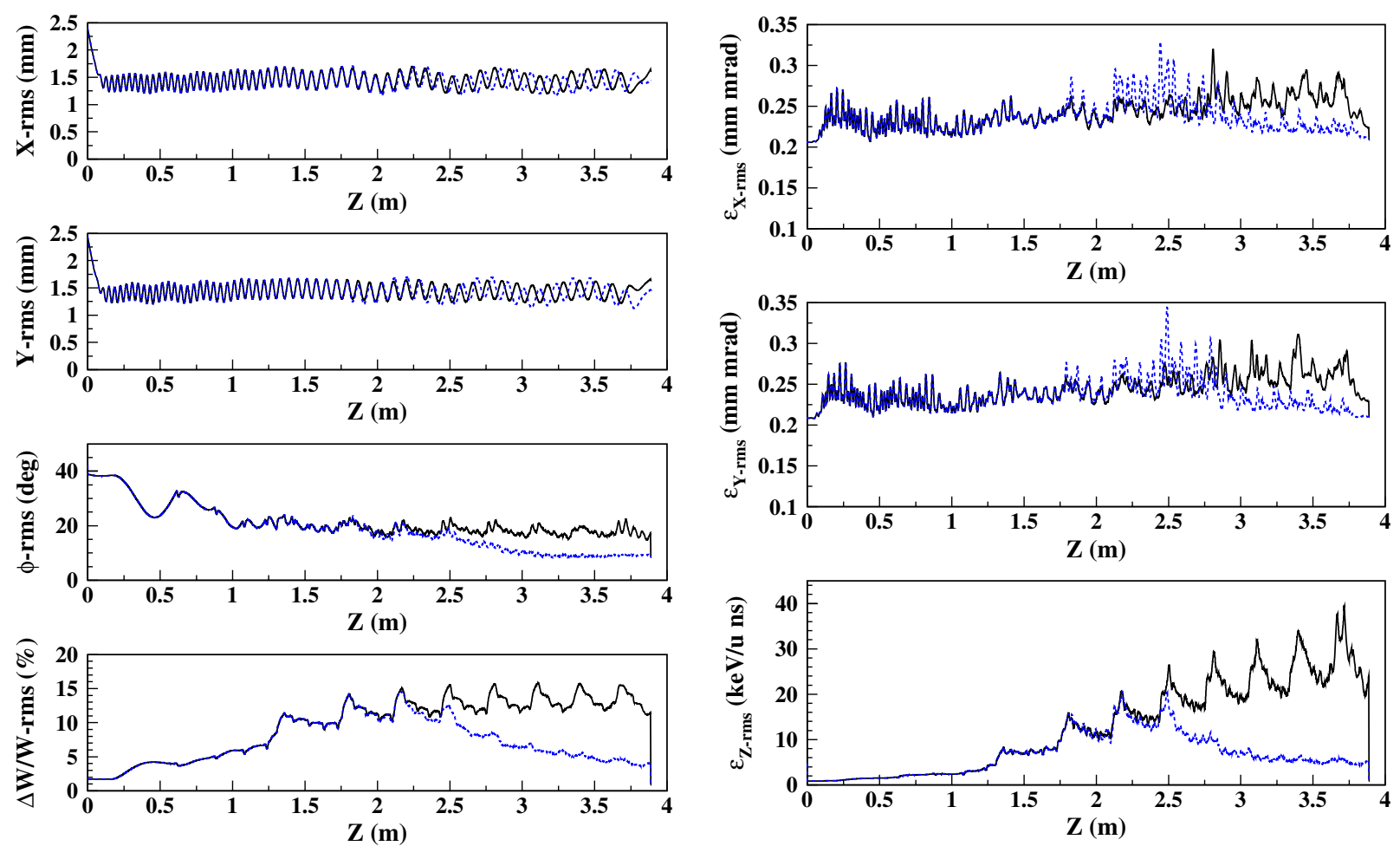

FIG. 11. Comparison between the original sinusoidal design (solid black) and the final trapezoidal design (dashed blue). On the left are rms beam envelopes and on the right are rms emittances along the RFQ. The curves include all particles within the aperture (accelerated or not). The peaks and valleys on the longitudinal plots, corresponding to oscillations of nonaccelerated particles, continue to develop in the sinusoidal design but they stop in the trapezoidal design due to filtering of the longitudinal tail discussed in the text.

parameters. The comparison of the total beam transmission confirms that a significant part of the beam is filtered by the trapezoidal vanes, which happened to be only the energy tail because the acceleration efficiency is the same in both cases. The acceleration efficiency is defined by an artificial $20 \%$ energy filter placed at the RFQ exit. Table $\mathrm{V}$ also shows that no emittance growth is observed for the rms values or for $99 \%$ of the beam. In conclusion, the narrower transverse acceptance of the trapezoidal design helped reject a significant part of the unwanted energy tail while transmitting $100 \%$ of the accelerated particles.

TABLE V. Comparison between the original sinusoidal and final trapezoidal modulation design for the ATLAS upgrade RFQ for the same power and length.

\begin{tabular}{lcc}
\hline \hline Parameter & Sinusoidal & Trapezoidal \\
\hline Output energy $(\mathrm{keV} / \mathrm{u})$ & 261.9 & 296.4 \\
Total transmission $(\%)$ & 97.2 & 89.3 \\
Accel. efficiency $(\%)$ & 82.7 & 82.7 \\
$4 \times \varepsilon(t, \mathrm{rms})(\mathrm{mm} \mathrm{mrad})$ & 0.84 & 0.84 \\
$4 \times \varepsilon(z, \mathrm{rms})(\mathrm{keV} / \mathrm{u} \mathrm{ns})$ & 3.51 & 3.59 \\
$\varepsilon(t, 99 \%)(\mathrm{mm} \mathrm{mrad})$ & 1.43 & 1.35 \\
$\varepsilon(z, 99 \%)(\mathrm{keV} / \mathrm{u} \mathrm{ns})$ & 10.0 & 10.8 \\
\hline \hline
\end{tabular}

\section{Effect of nonvanishing field in the end gaps}

In the case of a full-vane geometry, there is no longitudinal field component in the gaps between the end flanges and the vanes. But in the case of vanes with window cuts, if the horizontal vanes' ends are full, the vertical vanes' ends are cut and vice versa as shown on Fig. 12. This creates a longitudinal potential difference that leads to a nonvanishing longitudinal field component in the gaps between the flanges and the vanes. The same effect also exists in

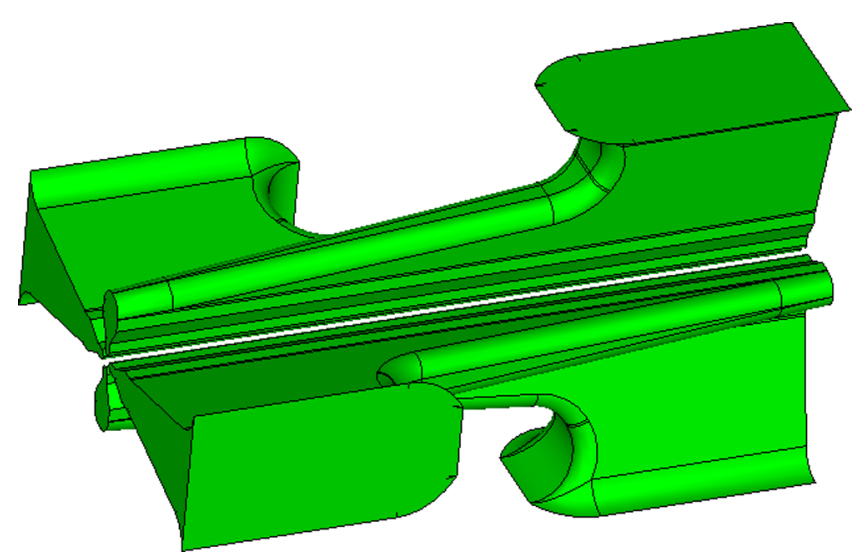

FIG. 12. MW-Studio model: RFQ vanes showing the alternating window cuts between the horizontal and vertical vanes. 

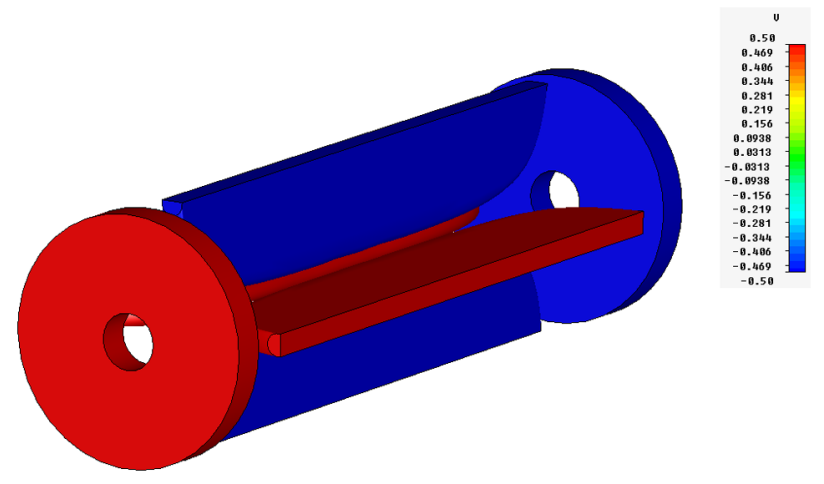

FIG. 13. EM-Studio model: Voltage applied to the vane tips and the flanges to simulate the longitudinal field in the gap between the flange and the vanes.

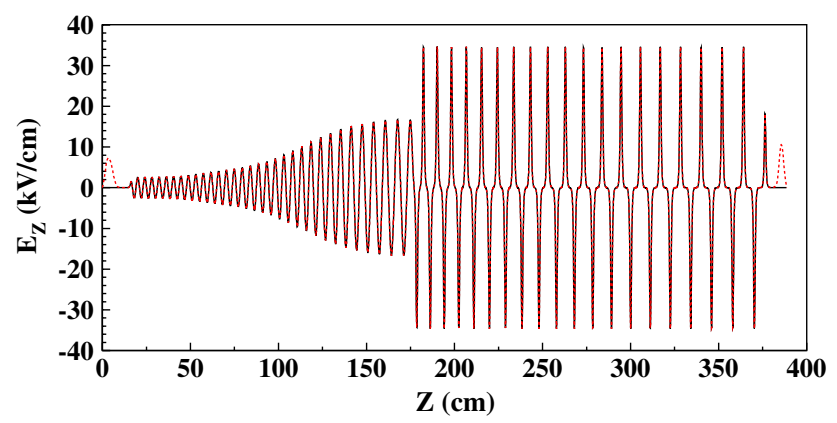

FIG. 14. Longitudinal field along the center of the RFQ before (solid black) and after (dashed red) including the field component in the gap between the flanges and the vanes.

four-rod RFQs. In MW-Studio this field component comes natural from the eigenmode solver but to simulate it in EM-Studio, we had to apply a voltage on the end flanges as shown in Fig. 13. The flange voltage should be the same as the full vane in that end.

Figure 14 shows a comparison of the longitudinal field along the RFQ before and after including the nonvanishing field in the gap. The effect of this field component on the output beam energy and beam emittances is summarized in Table VI. We notice that in our case, the longitudinal field component in the gap leads to a lower output energy.

TABLE VI. Effect of the nonzero longitudinal field in the gaps between the flanges and the vanes. Comparison of the RFQ output beam parameters before and after including this effect.

\begin{tabular}{lcc}
\hline \hline Parameter & No gap field & Gap field \\
\hline Output energy $(\mathrm{keV} / \mathrm{u})$ & 296.4 & 294.8 \\
Total transmission $(\%)$ & 89.3 & 88.4 \\
Accel. efficiency $(\%)$ & 82.7 & 81.9 \\
$4 \times \varepsilon(t, \mathrm{rms})(\mathrm{mm} \mathrm{mrad})$ & 0.84 & 0.84 \\
$4 \times \varepsilon(z, \mathrm{rms})(\mathrm{keV} / \mathrm{u} \mathrm{ns})$ & 3.59 & 3.64 \\
$\varepsilon(t, 99 \%)(\mathrm{mm} \mathrm{mrad})$ & 1.35 & 1.35 \\
$\varepsilon(z, 99 \%)(\mathrm{keV} / \mathrm{u} \mathrm{ns})$ & 10.8 & 11.8 \\
\hline \hline
\end{tabular}

TABLE VII. Effect of varying the input beam energy on the output beam parameters.

\begin{tabular}{lccc}
\hline \hline Input energy $(\mathrm{keV} / \mathrm{u})$ & 29.5 & 30.0 & 30.5 \\
\hline Output energy $(\mathrm{keV} / \mathrm{u})$ & 295.8 & 294.8 & 293.7 \\
Total transmission $(\%)$ & 81.4 & 88.4 & 89.9 \\
Accel. efficiency $(\%)$ & 75.2 & 81.9 & 82.7 \\
$4 \times \varepsilon(t, \mathrm{rms})(\mathrm{mm} \mathrm{mrad})$ & 0.86 & 0.84 & 0.86 \\
$4 \times \varepsilon(z, \mathrm{rms})(\mathrm{keV} / \mathrm{u} \mathrm{ns})$ & 4.33 & 3.64 & 3.55 \\
$\varepsilon(t, 99 \%)(\mathrm{mm} \mathrm{mrad})$ & 1.51 & 1.35 & 1.44 \\
$\varepsilon(z, 99 \%)(\mathrm{keV} / \mathrm{u} \mathrm{ns})$ & 15.9 & 11.8 & 10.9 \\
\hline \hline
\end{tabular}

In principle, it should be possible to redesign the end sections to end up with higher energy if desired or to correct for this effect to have the exact design output energy. This is important in the case where the RFQ is followed by a drift-tube linac designed for a given beta value, but in our case the RFQ is followed by a superconducting linac, so it does not require any energy correction to this effect. Table VI also shows that the rms emittances are practically the same but we notice a lower beam capture and an $\sim 10 \%$ growth in the $99 \%$ longitudinal emittance. The main reason for the longitudinal emittance growth is the longitudinal field in the entrance gap that affects both the synchronism and the bunching in the rest of the RFQ. A possible solution would be to change the input beam energy around the nominal value of $30 \mathrm{keV} / \mathrm{u}$. Table VII shows a comparison between three input energies; $29.5,30$, and $30.5 \mathrm{keV} / \mathrm{u}$. We clearly see that although it leads to lower output energy, a $30.5 \mathrm{keV} / \mathrm{u}$ input energy seems to correct the synchronism, reducing the bunch centroid oscillations caused by the entrance field gap and restoring the beam capture and longitudinal emittance to their original values. Therefore, $30.5 \mathrm{keV} / \mathrm{u}$ is now the official input energy for routine operations of the ATLAS RFQ.

\section{E. Effect of aperture model choice}

Depending on the beam current and the matching, the beam could be very well confined close to the beam axis away from the vanes or large with tails approaching the vanes. A good RFQ should be designed and operated in the first configuration; in this case the aperture model should not affect the calculation of the RFQ transmission. It is, however, important to consider the vane shape as a more realistic aperture model. We here use three different aperture models and calculated the corresponding RFQ transmission for comparison. The first model uses the average radius $r_{0}$ all over the RFQ as the aperture; the second uses the cells apertures, $a=2 \times r_{0} /(m+1)$, where $m$ is the cell modulation. The last model uses the actual vane shape. Table VIII shows the results for both the trapezoidal and sinusoidal models. We clearly see that the total transmission is more affected by the choice of the aperture model, 
TABLE VIII. Effect of the choice of the aperture model on the calculation of the RFQ transmission for both the sinusoidal and trapezoidal designs. $T$ is the total transmission and $A$ is the acceleration efficiency.

\begin{tabular}{lllll}
\hline \hline & \multicolumn{2}{c}{ Sinusoidal } & \multicolumn{2}{c}{ Trapezoidal } \\
Aperture model & $\mathrm{T}(\%)$ & $\mathrm{A}(\%)$ & $\mathrm{T}(\%)$ & $\mathrm{A}(\%)$ \\
\hline Average radius $\left(r_{0}\right)$ & 98.0 & 82.7 & 89.9 & 82.7 \\
$\begin{array}{l}\text { Cells' apertures } \\
\quad\left(a=2 \times r_{0} /(m+1)\right)\end{array}$ & 96.9 & 82.2 & 89.1 & 82.2 \\
$\quad$ Vane modulation & 98.7 & 82.7 & 89.6 & 82.5 \\
\hline \hline
\end{tabular}

while the acceleration efficiency is practically the same for all the models within a $0.5 \%$ error.

\section{F. Effect of vane modulation errors}

No prototype was built for the current RFQ. The fabrication procedure was successfully tested a few years ago to build a one-segment $57.5 \mathrm{MHz}$ prototype for the rare isotope accelerator RFQ [30]. The RF performance of the prototype was successfully reproduced by the simulations [31], which constituted an important benchmark for the 3D approach used for this RFQ. However, a beam test was not performed to check the vane modulation on the prototype. To test this critical part of the fabrication, which is essential for the beam dynamics especially with the transition from sinusoidal to trapezoidal modulation, we have built aluminum models of the vane tips on which the modulation was applied using the same machine to be used for the real copper vanes. Figure 15 shows pictures of the aluminum vane tip models. Coordinate measuring machine measurements were performed on these models and Fig. 16 shows a comparison between the input data used to apply the vane modulation and data points measured on the actual models. We can see an average deviation of about $20 \mu \mathrm{m}$ with extreme values of about $40 \mu \mathrm{m}$. Because of the difference in material properties between aluminum and copper, the modulation precision
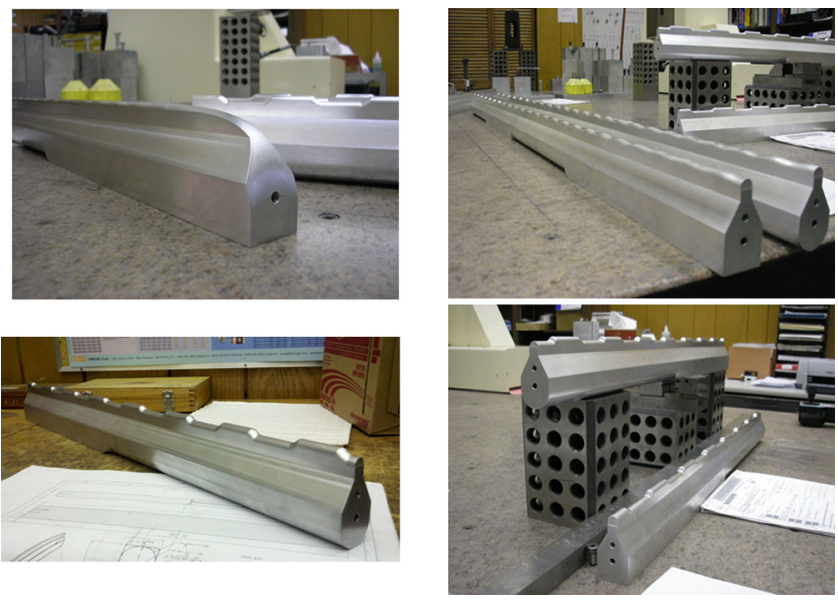

FIG. 15. Aluminum models of vane tip modulation for all RFQ segments.
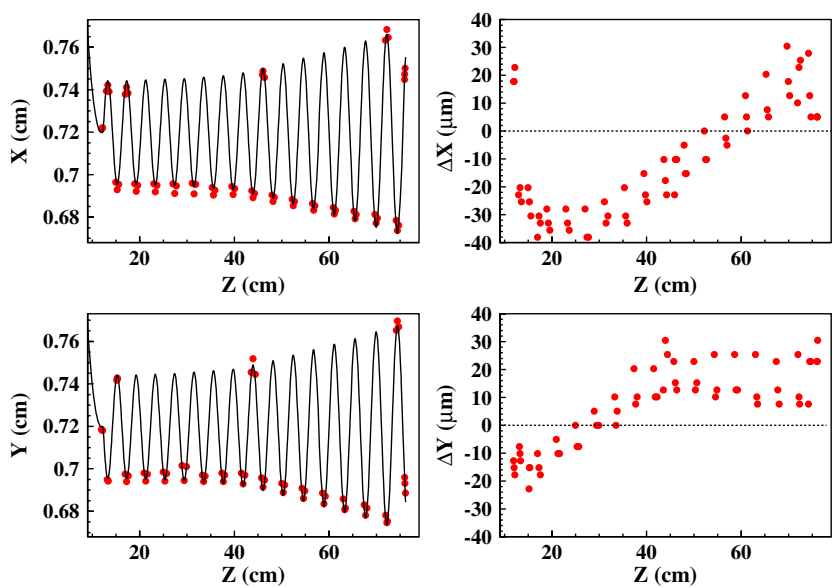

FIG. 16. Comparison of calculated vane profile (curves) and measured data points (dots) on the actual aluminum model (left) and corresponding deviations in $\mu \mathrm{m}$ (right).

was expected to be better on copper, which was confirmed on the final RFQ to not exceed $25 \mu \mathrm{m}$.

In order to simulate the effect of modulation precision errors on the beam dynamics, we have simulated the full RFQ structure in MWS with different error types and amplitudes. The extracted 3D fields for every case are then used to track the beam and check its output parameters. The extracted fields from MWS also include the effect of field detuning caused by moving the vanes. Table IX shows the effect of a global symmetric shift of the vanes away from each other with increasing distance. Table $\mathrm{X}$ shows the effect of random errors on the modulation with increasing amplitudes and Table XI shows the combined

TABLE IX. Effect of global shifts of the vanes from each other with increasing distance.

\begin{tabular}{lrrrr}
\hline \hline Shift $(\mu \mathrm{m})$ & \multicolumn{1}{c}{0} & \multicolumn{1}{c}{25} & 50 & 100 \\
\hline Output energy $(\mathrm{keV} / \mathrm{u})$ & 294.3 & 294.1 & 294.4 & 294.0 \\
Transmission $(\%)$ & 82.7 & 82.6 & 82.5 & 82.5 \\
$4 \times \varepsilon(t, \mathrm{rms})(\mathrm{mm} \mathrm{mrad})$ & 0.86 & 0.88 & 0.86 & 0.87 \\
$4 \times \varepsilon(z, \mathrm{rms})(\mathrm{keV} / \mathrm{u} \mathrm{ns})$ & 3.45 & 3.38 & 3.36 & 3.31 \\
$\varepsilon(t, 99 \%)(\mathrm{mm} \mathrm{mrad})$ & 1.38 & 1.47 & 1.40 & 1.41 \\
$\varepsilon(z, 99 \%)(\mathrm{keV} / \mathrm{u} \mathrm{ns})$ & 9.34 & 9.06 & 8.88 & 8.76 \\
\hline \hline
\end{tabular}

TABLE X. Effect of random vane modulation error with increasing amplitude.

\begin{tabular}{lrrrr}
\hline \hline Random $(\mu \mathrm{m})$ & 0 & 25 & 50 & 100 \\
\hline Output energy $(\mathrm{keV} / \mathrm{u})$ & 294.3 & 292.9 & 292.2 & 292.4 \\
Transmission $(\%)$ & 82.7 & 83.1 & 83.1 & 83.0 \\
$4 \times \varepsilon(t, \mathrm{rms})(\mathrm{mm} \mathrm{mrad})$ & 0.86 & 0.86 & 0.88 & 0.84 \\
$4 \times \varepsilon(z, \mathrm{rms})(\mathrm{keV} / \mathrm{u} \mathrm{ns})$ & 3.45 & 3.94 & 3.89 & 3.87 \\
$\varepsilon(t, 99 \%)(\mathrm{mm} \mathrm{mrad})$ & 1.38 & 1.38 & 1.46 & 1.31 \\
$\varepsilon(z, 99 \%)(\mathrm{keV} / \mathrm{u} \mathrm{ns})$ & 9.34 & 11.70 & 12.16 & 12.68 \\
\hline \hline
\end{tabular}


TABLE XI. Combined effect of global shifts and random vane modulation errors with increasing amplitudes.

\begin{tabular}{lcccc}
\hline \hline & 0 & 25 & 50 & 100 \\
Shift and random $(\mu \mathrm{m})$ & and 0 & and 25 & and 50 & and 100 \\
\hline Output energy $(\mathrm{keV} / \mathrm{u})$ & 294.3 & 293.1 & 293.3 & 293.6 \\
Transmission $(\%)$ & 82.7 & 83.1 & 83.1 & 82.9 \\
$4 \times \varepsilon(t, \mathrm{rms})(\mathrm{mm} \mathrm{mrad})$ & 0.86 & 0.86 & 0.85 & 0.86 \\
$4 \times \varepsilon(z, \mathrm{rms})(\mathrm{keV} / \mathrm{u} \mathrm{ns})$ & 3.45 & 4.11 & 3.72 & 4.88 \\
$\varepsilon(t, 99 \%)(\mathrm{mm} \mathrm{mrad})$ & 1.38 & 1.39 & 1.33 & 1.40 \\
$\varepsilon(z, 99 \%)(\mathrm{keV} / \mathrm{u} \mathrm{ns})$ & 9.34 & 11.95 & 11.21 & 12.40 \\
\hline \hline
\end{tabular}

effect of global shifts and random modulation errors. Shifting the vanes from each other up to $100 \mu \mathrm{m}$ does not seem to affect the energy and the transmission significantly, but it seems to have consistently reduced the longitudinal emittance somewhat equivalent to a slight reduction in vane voltage. However, the transverse emittance growth is not consistent as we expect from the reduction in focusing. From Table X, a random error applied to the vane modulation disturbs the synchronism between the beam and the field leading to a more significant change in energy and transmission as well as consistent emittance growth with increasing error amplitude. We also notice a transverse emittance growth that does not seem to be consistent. Combining a global vane shift with a random error on the modulation in Table XI shows similar effects as those observed with the random error only but with more fluctuations. In summary, a global vane shift of up to $100 \mu \mathrm{m}$ does not seem to affect the beam significantly while a similar random error on the vane modulation reduces the energy and enhances the longitudinal beam tail. We should mention that these studies are hypothetical and the closest case to the ATLAS RFQ would be the $25 \mu \mathrm{m}$ random vane modulation error (Table $\mathrm{X}$, column 2 ).

\section{ELECTROMAGNETIC DESIGN AND SIMULATIONS}

The main goals of the electromagnetic (EM) design are to produce a design with the lowest RF power consumption, a target frequency tunable to the operational frequency, and a flat intervane field along the RFQ. The ATLAS RFQ design was based on the original $57 \mathrm{MHz}$ rare isotope accelerator RFQ [22] with similar power requirements. To control the frequency and field flatness, we have studied the effects of the modulation and the tuners, as well as the higher-order modes and their separation from the operational mode. For this purpose our frequency and field calculations have to be accurate and reproducible especially when including the modulations.

The electromagnetic design was performed using MWS from the CST simulation package [13]. The original single vane model was imported into MWS from the Pro/Engineer CAD software [32] used to produce all the official drawings. The full four-vane structure is built by copying and mirroring the original single vane. The vane modulation is applied cell by cell as described in [31]. An octagonal RF volume is defined from which the solid vanes are extracted to keep only the vacuum volume.

Because of the level of detail in the full RFQ model, the mesh choice for the EM simulations becomes very important. In our previous study [31] we have shown that using a finer local mesh around the vane tip area, while leaving the rest of the structure for automatic meshing in MWS, produced a more accurate frequency when compared to the measured value for a prototype $57 \mathrm{MHz}$ RFQ. In addition to the original hexahedral mesh option, we have repeated the simulations using a higher-order tetrahedral mesh option that has become available in the recent versions of CST. A typical electromagnetic simulation of the full structure including all the details takes about $24 \mathrm{~h}$ on a 32-core machine with 256 GB of memory.

\section{A. Frequency calculation}

We have previously estimated the error on the calculated frequency in MWS to be about $200 \mathrm{kHz}$ using the hexahedral mesh [31]; for this reason, we lowered the target frequency from $60.4 \mathrm{MHz}$ to $60.25 \mathrm{MHz}$ with a full tuning range of $800 \mathrm{kHz}$ centered around the operational frequency of $60.625 \mathrm{MHz}$. Table XII shows the frequency before and after applying the vane modulation obtained with the original hexahedral mesh and the new tetrahedral mesh.

We clearly notice the modulation shifting the frequency up by about $500 \mathrm{kHz}$. We also notice that the tetrahedral mesh result suggests a lower frequency by about $100 \mathrm{kHz}$, which seems to agree better with the measured frequency on the individual segments as well as the full RFQ as shown in Table XIII. The segments' frequencies were

TABLE XII. RFQ frequency calculated with different mesh options before and after including the vane modulation.

\begin{tabular}{lcc}
\hline \hline Geometry & Hexahedral mesh & Tetrahedral mesh \\
\hline No modulation & $59.77 \mathrm{MHz}$ & $59.64 \mathrm{MHz}$ \\
Modulated vanes & $60.27 \mathrm{MHz}$ & $60.15 \mathrm{MHz}$ \\
\hline \hline
\end{tabular}

TABLE XIII. Comparison of calculated and measured frequencies for individual RFQ segments and the full RFQ. The calculations are performed using the tetrahedral mesh.

\begin{tabular}{lccc}
\hline \hline Geometry & $\begin{array}{c}\text { Calculated } \\
f(\mathrm{MHz})\end{array}$ & $\begin{array}{c}\text { Measured } \\
f(\mathrm{MHz})\end{array}$ & $\begin{array}{c}\text { Difference } \\
\Delta f(\mathrm{kHz})\end{array}$ \\
\hline Segment \#1 & 58.78 & 58.82 & 30 \\
Segment \#2 & 57.97 & 58.06 & 90 \\
Segment \#3 & 58.16 & 58.19 & 30 \\
Segment \#4 & 58.26 & 58.24 & 20 \\
Segment \#5 & 58.88 & 58.85 & 30 \\
Full RFQ & 60.15 & 60.06 & 90 \\
\hline \hline
\end{tabular}


measured before brazing while the full RFQ frequency was measured after brazing.

We notice that the calculated frequency is consistently higher than the measured one with a maximum deviation of about $100 \mathrm{kHz}$. The $200 \mathrm{kHz}$ difference between the measured frequency and the originally calculated frequency of 60.27 MHz using the hexahedral mesh confirms our estimate of the calculation error. The measured frequency is lower but remains within the designed tuning range. With better description of the geometry curvature, the new highorder tetrahedral mesh seems to reproduce better the measured frequency and will be used for future RFQ design.

\section{B. Neighboring modes}

In addition to the operating mode, a RFQ could resonate at different modes that may interfere with the main mode if their frequencies are very close. To calculate all excitation modes within a given frequency range, we have to disable the symmetry option in the calculation, which significantly reduces the effective number of mesh cells in the simulation and hence the accuracy of the results. Since we are interested mainly in the frequency separation of these modes, we may simplify the problem by not including the vane modulation. We know the frequency shift from the vane modulation, which should not affect the relative mode frequency separation. Table XIV presents the results of these simulations for the first three modes.

We notice that the main mode comes first and the next mode is separated by more than $10 \mathrm{MHz}$. This is a distinguished feature of the split-coaxial or window-coupled structure chosen for this RFQ. In addition to reducing the transverse size of the RFQ, the windows in the vanes provide strong coupling between the segments, which leads to good mode separation and less field sensitivity to tuners. These two characteristics are very important for stable operation of the RFQ.

\section{Modulation effect}

We have performed a detailed study of the effects of the different parts of the vane modulation on the RFQ frequency and field flatness. The results are reported in Table XV for the frequency and Fig. 17 for the field flatness. We notice that before applying any modulation the field has a dip around the center of the RFQ. Adding the input matcher only, moves the dip to the entrance creating a positive field slope as a function of $Z$, while adding only

TABLE XIV. Types and frequencies of the first 3 RFQ modes calculated without including the vane modulation.

\begin{tabular}{llc}
\hline \hline Mode & Type & Frequency $(\mathrm{MHz})$ \\
\hline 1 & quadrupole & 59.60 \\
2 & quadrupole & 70.13 \\
3 & dipole & 89.20 \\
\hline \hline
\end{tabular}

TABLE XV. Effect of the different parts of vane modulation on the frequency.

\begin{tabular}{lcc}
\hline \hline Effect & Frequency $(\mathrm{MHz})$ & Shift $(\mathrm{kHz})$ \\
\hline No modulation & 59.64 & reference \\
Input matcher & 59.85 & 210 \\
Output matcher & 59.77 & 130 \\
Input and output & 59.99 & 350 \\
Full modulation & 60.15 & 510 \\
\hline \hline
\end{tabular}

the output matcher does the opposite. The tilt in the field comes from the difference in capacitance between both ends of the RFQ. Including both the input and output matchers reduces the slope while applying the full modulation reverses the slope but keeps it at about $2 \%$. For the frequency, the results are consistent with the values obtained previously using the hexahedral mesh.

\section{Tuners effect}

The RFQ was designed with 12 tuners, each of them is $5.2 \mathrm{in}$. in diameter and could be inserted $2 \mathrm{in}$. deep into the RFQ volume allowing a tuning range of about $800 \mathrm{kHz}$. Figure 18 shows the effect of the tuner's depth on the

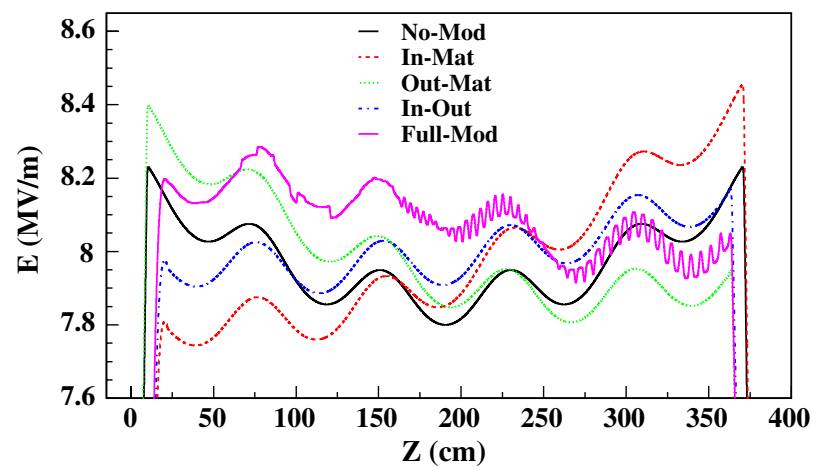

FIG. 17. Intervane electric field along the RFQ for different components of the modulation.

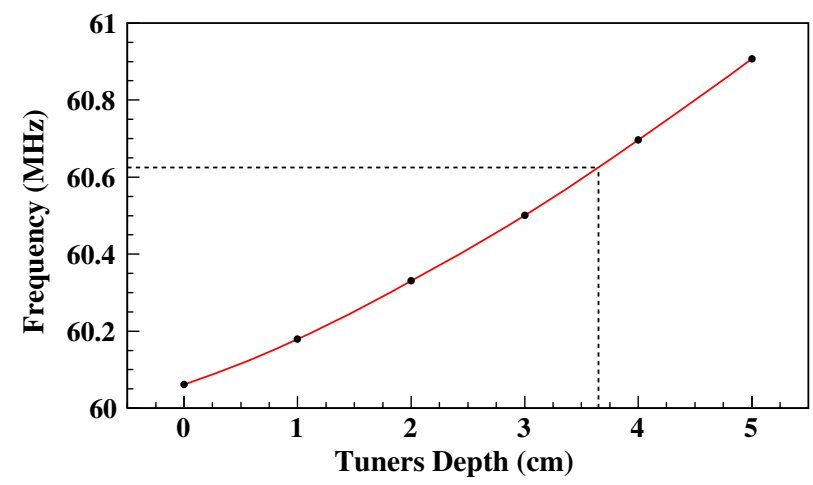

FIG. 18. Calculated RFQ frequency as function of uniform tuners depth. The dashed lines point to the operating frequency and the required depth to tune it. 


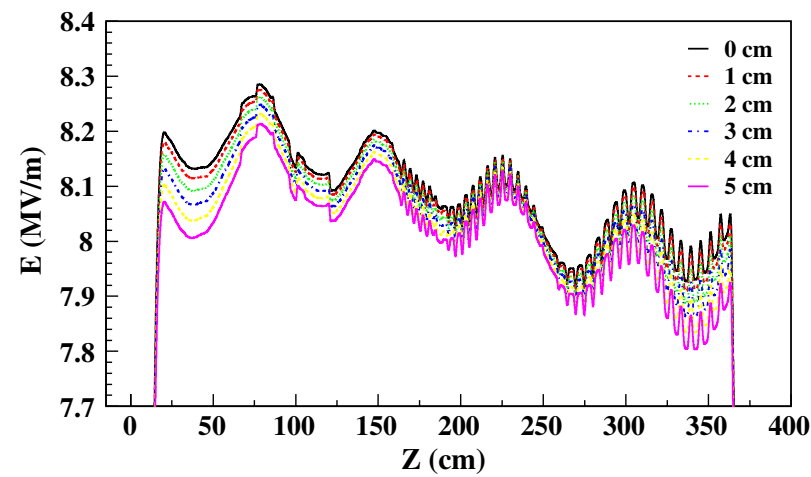

FIG. 19. Intervane electric field along the RFQ for different uniform tuner's depth.

frequency when inserted uniformly inside the RFQ. To tune the RFQ from the measured frequency of $60.056 \mathrm{MHz}$ to the operational frequency of $60.625 \mathrm{MHz}$, the required uniform tuner's depth is about $3.5 \mathrm{~cm}$ or $1.4 \mathrm{in}$. Experimentally and considering the fact that the tuners are not uniformly distributed among the segments, the operating frequency was reached by inserting the three tuners in each of the first and last segments to $1 \mathrm{in}$. in depth and the two tuners in each of the other segments to $1.75 \mathrm{in}$. in depth, for an average depth of 1.375 in., which is very close to the calculated value of $1.4 \mathrm{in}$. Figure 19 shows the effect of the tuners depth on the intervane field along the RFQ. Local averaging was applied to all curves to smooth out the local effect of the modulation and emphasize the large scale effect along the RFQ. We see clearly that the field is barely affected by the tuner's depth; the field slope is about $2 \%$ with variations below $1 \%$. As mentioned above, for actual operations the tuners were not inserted uniformly. Figure 20 shows a comparison of the intervane field between the actual configuration and a uniform tuner's depth to tune to the same frequency. Again the effect is in the order of $1 \%$ and should not affect the output beam parameters. This field insensitivity to the tuners is mainly

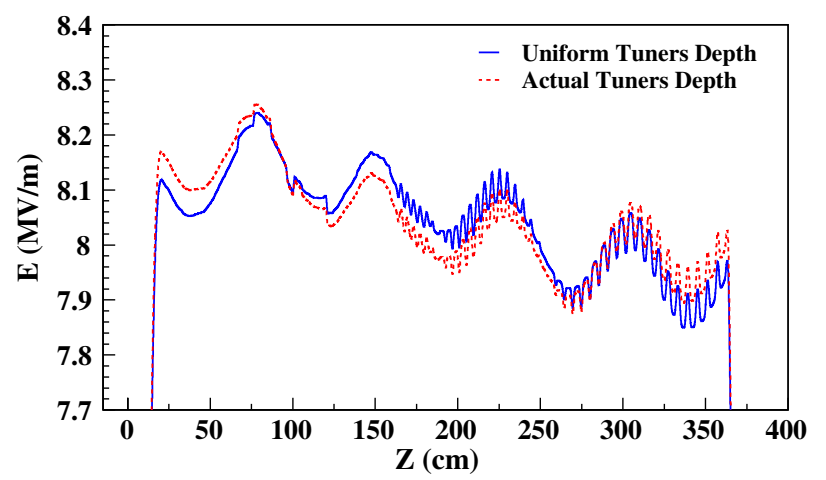

FIG. 20. Intervane electric field along the RFQ for the actual tuner's configuration and the equivalent uniform tuner's depth $(3.5 \mathrm{~cm})$.
TABLE XVI. Peak surface electric field values calculated with both EM-Studio and MW-Studio for both the sinusoidal and trapezoidal designs.

\begin{tabular}{lccc}
\hline \hline Design & EM-Studio & MW-Studio & Units \\
\hline Sinusoidal & 1.32 & 1.42 & Kilpatrick \\
Trapezoidal & 1.61 & 1.67 & Kilpatrick \\
\hline \hline
\end{tabular}

due to the strongly coupled nature of the window-coupled structure of this RFQ.

\section{E. Peak fields: trapezoidal versus sinusoidal}

The trapezoidal modulation involves long straight sections and smaller radii of curvature than the sinusoidal one (see Fig. 3) which may lead to higher peak surface fields. For this purpose, we have simulated both designs in both EM-Studio and MW-Studio; Table XVI shows the results. The Kilpatrick field limit at this frequency is about $9.5 \mathrm{MV} / \mathrm{m}$. Changing the modulation from sinusoidal to trapezoidal increased the peak surface electric field by about $20 \%$, that is from $\sim 1.4$ to $\sim 1.7$ Kilpatrick units, which remains acceptable. Figure 21 shows clearly that the field is more uniformly distributed in the case of sinusoidal modulation while higher surface fields are localized on the tips of the curved sections in the trapezoidal modulation.
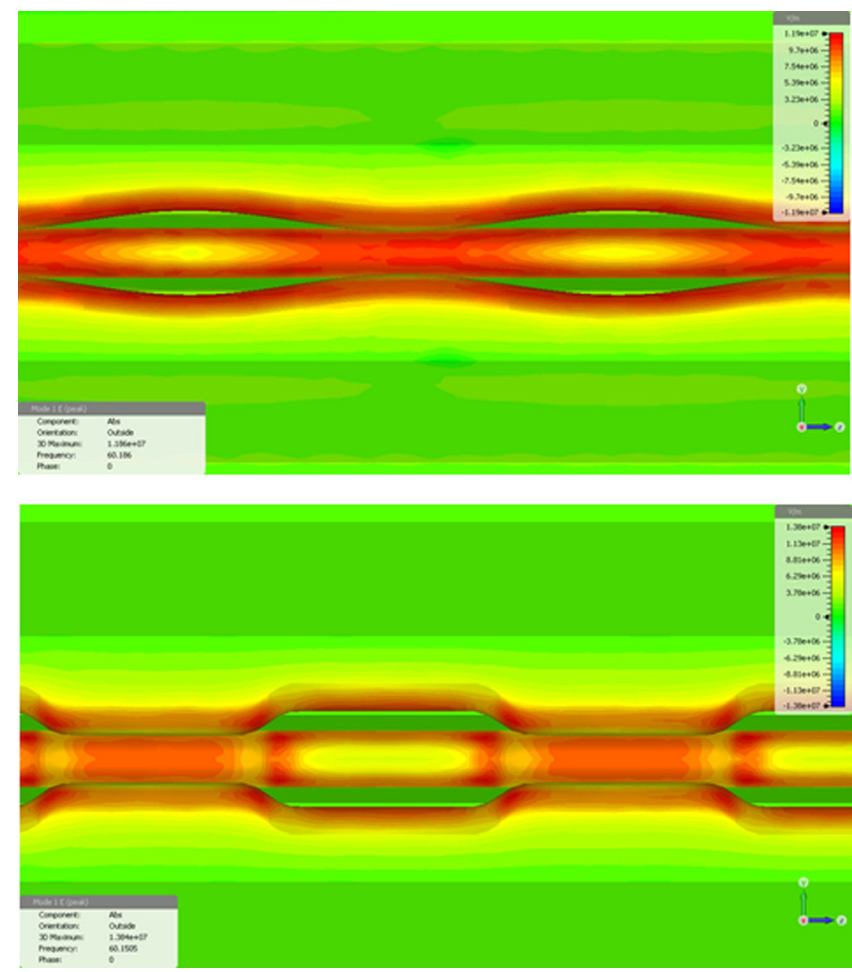

FIG. 21. Peak surface electric fields calculated in MW-Studio for both the sinusoidal (top) and trapezoidal (bottom) RFQ designs. 


\section{CONCLUSIONS}

The full 3D methods developed in this work were made possible by the significant advancements in computing capabilities and the accuracy of electromagnetic design software. The new approach combines both the beam dynamics and the electromagnetic design in a single process. It was successfully applied to the design and simulations of the ATLAS upgrade RFQ, which has shown a great performance in routine ATLAS operations. The ATLAS RFQ is the first cw RFQ with trapezoidal modulations in the accelerating section. A new type of output radial matcher was developed to produce an axial-symmetric beam at the RFQ exit for direct injection into a solenoidal focusing channel. The methods developed here should be applicable to any RFQ design. Comparisons to the experimental data show a very good agreement with the simulations' results. No prototype was built for the ATLAS RFQ and no bead pull measurements were performed prior to beam tests, yet the RFQ performed exceptionally well from the first day. This suggests that detailed 3D simulations have become more reliable and could perhaps replace the prototyping phase to build the final product at once, although we recognize that prototyping may still be needed to establish and validate the fabrication procedure.

\section{ACKNOWLEDGMENTS}

The authors would like to thank A. Barcikowski and G. Zinkann for the frequency measurements on the individual segments and the full ATLAS upgrade RFQ, and Z. Conway for reading and discussing the manuscript. This work was supported by the U.S. Department of Energy, Office of Nuclear Physics, under Contract No. DE-AC02$06 \mathrm{CH} 11357$.

[1] I. M. Kapchinsky and V. A. Teplyakov, Prib. Tekh. Eksp. 2, 19 (1970).

[2] V. A. Teplyakov and A.P. Maltsev, Ion Linear Accelerators with RF Quadrupole Focusing in IHEP, News and Problems of Fundamnetal Physics, Vol. 2 (IHEP, Protvino, 2008) (in Russian).

[3] I. M. Kapchinsky, Theory of Resonance Linear Accelerators (Harwood, Chur, Switzerland, 1985).

[4] L. Young, Proceedings of the 20th Particle Accelerator Conference, PAC-2003, Portland, OR, 2003 (IEEE, New York, 2003), p 60.

[5] C. Rossi et al., Proceedings of the 4th International Particle Accelerator Conference, IPAC-2013, Shanghai, China, 2013, (JACoW, Shanghai, China, 2013) p. 3951.

[6] P. N. Ostroumov et al., Phys. Rev. ST Accel. Beams 15, 110101 (2012).

[7] A. Perry, C. Dickerson, P. N. Ostroumov, and G. Zinkann, Nucl. Instrum. Methods Phys. Res., Sect. A 735, 163 (2014).
[8] P. N. Ostroumov et al., Proceedings of the 25th Particle Accelerator Conference, PAC-2013, Pasadena, CA, 2013, (IEEE, New York, 2013), paper WEOAB2.

[9] Parmteq Manual, Los Alamos Report No. LA-UR-96-1836.

[10] A. A. Kolomiets, T. E. Tretjakova, and S. G. Yaramishev, "DESRFQ - a Code for Generation of Radio Frequency Quadrupole," ITEP-Moscow internal report, 1998.

[11] Superfish Manual, Los Alamos Report No. LA-UR-961834.

[12] M. Clemens et al., Proceedings of the 1999 International Conference of Computational Electromagnetics and Its Applications, ICCEA '99, Beijing, China, 1999 (IEEE, New York, 1999), p. 565.

[13] CST simulation packages, www.cst.com.

[14] P. N. Ostroumov et al., Proceedings of the 2011 Particle Accelerator Conference, PAC-2011, New York, NY, 2011 (IEEE, New York, 2011), p. 2110.

[15] R. W. Muller, GSI Report No. 89-04, 1989.

[16] S. Arai et al., Proceedings of the 11th Particle Accelerator Conference, PAC-1985, Vancouver, BC, Canada, 1985 (IEEE, New York, 1985), p. 3175.

[17] V.A. Andreev and G. Parisi, Proceedings of the 15th Particle Accelerator Conference, PAC-1993, Washington, DC, 1993 (IEEE, New York, 1993), p. 3124.

[18] T. Schultheiss (private communication).

[19] T. Schultheiss et al., Proceedings of the 2011 Particle Accelerator Conference, PAC-2011, New York, NY, 2011 (IEEE, New York, 2011), p. 1334.

[20] J. W. Staples, Proceedings of the 17th International Linac Conference, LINAC-1994, Tsukuba, Japan, 1994 (KEK, Tsukuba, Japan, 1994), p. 755.

[21] P.N. Ostroumov et al., Proceedings of the 20th International Linac Conference, LINAC-2000, Monterey, CA, 2000 (SLAC, Menlo Park, CA, 2000), p. 202.

[22] P. N. Ostroumov, A. Kolomiets, D. Kashinsky, S. Minaev, V. Pershin, T. Tretyakova, and S. Yaramishev, Phys. Rev. ST Accel. Beams 5, 060101 (2002).

[23] B. Mustapha et al., Proceedings of the 22nd Particle Accelerator Conference, PAC-2007, Albuquerque, New Mexico, 2007 (IEEE, New York, 2007), p. 1661.

[24] K. R. Crandall, Proceedings of the 12th International Linac Conference, LINAC-1984, Seeheim, Germany, 1984 (GSI, Darmstadt, Germany, 1984), p. 109.

[25] P. N. Ostroumov and V. N. Aseev, JINST 1, P04002 (2006).

[26] B. Mustapha et al., Proceedings of the 25th International Linear Accelerator Conference, LINAC-2010, Tsukuba, Japan (KEK, Tsukuba, Japan, 2010), p. 542.

[27] O. K. Belyaev et al., Proceedings of the 20th International Linac Conference, LINAC-2000, Monterey, CA, 2000 (SLAC, Menlo Park, CA, 2000), p. 259.

[28] K. R. Crandall, Los Alamos Report No. LA-9695-MS.

[29] The beam dynamics code TRACK, www.phy.anl.gov/ atlas/TRACK.

[30] P. N. Ostroumov et al., Proceedings of the 23rd International Linac Conference, LINAC-2006, Knoxville, TN, 2006 (JACoW, Knoxville, TN, 2006), p. 767.

[31] B. Mustapha et al., Proceedings of the 2011 Particle Accelerator Conference, PAC-2011, New York, NY, 2011 (IEEE, New York, 2011), p. 97.

[32] Pro/Engineer CAD software, www.ptc.com. 